\title{
Activation of Anti-tumor Immune Response by Ablation of HCC with Nanosecond Pulsed Electric Field
}

\author{
Xiaobo Xu ${ }^{\# 1}$, Yiling Chen ${ }^{\# 2}$, Ruiqing Zhang ${ }^{3}$, Xudong Miao ${ }^{4}$ and Xinhua Chen*1 \\ ${ }^{1}$ Collaborative Innovation Center for Diagnosis Treatment of Infectious Diseases, Key Laboratory of Combined Multi-organ \\ Transplantation, Ministry of Public Health, Department of Hepatobiliary and Pancreatic Surgery, First Affiliated Hospital, Zhejiang \\ University, Hangzhou, Zhejiang, China; ${ }^{2}$ Department of Anatomy, Tianjin Medical University, Tianjin, China; ${ }^{3}$ Hepatobiliary \& \\ Hydatid Department, Digestive and Vascular Surgery Centre, Xinjiang Key Laboratory of Echinococcosis, First Affiliated Hospital of \\ Xinjiang Medical University, Urumqi, China; ${ }^{4}$ Department of Orthopedics, Second Affiliated Hospital, Zhejiang University, \\ Hangzhou, Zhejiang, China
}

\begin{abstract}
Locoregional therapy is playing an increasingly important role in the non-surgical management of hepatocellular carcinoma (HCC). The novel technique of non-thermal electric ablation by nanosecond pulsed electric field has been recognized as a potential locoregional methodology for indicated HCC. This manuscript explores the most recent studies to indicate its unique anti-tumor immune response. The possible immune mechanism, termed as nano-pulse stimulation, was also analyzed.

Citation of this article: Xu X, Chen Y, Zhang R, Miao X, Chen $X$. Activation of anti-tumor immune response by ablation of HCC with nanosecond pulsed electric field. J Clin Transl Hepatol 2018;6(1):85-88. doi: 10.14218/JCTH.2017.00042.
\end{abstract}

\section{Introduction}

Locoregional therapy refers to the locally applied minimally invasive interventional procedures to destroy tumors directly. It is guided by a radiological method to insert the ablation device precisely, with or without combination of chemomedicine to enhance tumor death. With the benefit of the tumor being treated locally, it has played an increasingly important role in the non-surgical management of hepatocellular carcinoma (HCC). Radiofrequency ablation (RFA) therapy, one of the locoregional therapies, has been recognized as producing effect comparable to surgery for small HCC. ${ }^{1}$

The novel electric ablation by nanosecond pulsed electric field (nSPEF) ablates tumors by non-thermal effects, without combining any chemo-therapeutics. ${ }^{2}$ The nsPEF technique has an advantage over RFA in its being non-thermal. Therefore, nsPEF can eliminate tumor cells, without thermal injury, on the adjacent organ and vessels. Different from conventional

Keywords: Locoregional therapy; Hepatocellular carcinoma (HCC); Nanosecond pulsed electric field (nSPEF); Nano-pulse stimulation (NPS).

Abbreviations: $\mathrm{HCC}$, hepatocellular carcinoma; nsPEF, nanosecond pulsed electric field; NPS, nano-pulse stimulation; RFA, radiofrequency ablation.

Received: 20 June 2017; Revised: 1 September 2017; Accepted: 6 September 2017

\#These two authors contributed equally to this study.

* Correspondence to: Xinhua Chen, Department of Hepatobiliary and Pancreatic Surgery, First Affiliated Hospital Zhejiang University School of Medicine, 79 Qingchun Road, Hangzhou, Zhejiang 310003, China. Tel: +86-571-87236570, Fax: +86-571-87236466, E-mail: xinhua_chen@zju.edu.cn heat-based ablation techniques, however, nsPEF does not cause direct Joel heat accumulation in the targeted region but induces apoptosis. ${ }^{3,4}$ It has been applied in humans and approved according to its efficacy. ${ }^{5}$

Delivering a high voltage electric field in ultra-short pulses, nsPEF disrupts both the plasma membrane and intracellular structures, causing clean cell death. ${ }^{6}$ As demonstrated in a previous study, ${ }^{7}$ tumor cells treated by nsPEF in vitro did not show severe necrotic morphological changes. In contrast, tumor ablated by nsPEF underwent gradual shrinkage, with minimal bleeding or necrosis. ${ }^{8-10}$ The nsPEF also features a precisely targeted ablation area, achieved with proper electrical parameter setting and electrode design.

As a result, tissues of different conductivity (other than tumors) can be preserved. This process explains why some tumor locations with critical vessels, which were impossible to treat by thermal ablation, are now accessible for treatment. ${ }^{11}$ nSPEF also shows advantages when the pulse duration is shorter, the electric field strength is more intensive, and the energy released is more controllable. These features are, particularly significant for treating HCC near major vascular vessels. In a previous study, the strategies of applying nSPEF in either a single dose $\mathrm{e}^{12}$ or multiple fractionated doses were compared. ${ }^{13,14}$ The multiple fractionated dose of nsPEF was found to inhibit tumors more effectively than the single dose, implying that the immune system was involved in the tumor reaction. To address the hypothesis, the nsPEF treatment was applied to a highly metastatic HCC xenograft model and the effects were studied. The multi-fractionated dose group did not show increased pulmonary metastasis but achieved the same low metastasis rate as the surgery-treated group. ${ }^{14}$ This evidence supported that lung metastasis can be minimized by efficient ablation of the primary tumor by immune stimulation.

\section{nsPEF causes bio-effects}

With high power and low energy given in ultra-short pulses, nSPEF produced unique intracellular bio-effects. nsPEF ablation induced tumor cell apoptosis and caused high-density nanopores to form in plasma membranes. The destroyed tumor cells released signals (e.g. cytokines, interleukins and chemokines) which trigger the host immune stimulation. Subsequently, the ablation zone also attracts macrophage infiltration and $\mathrm{T}$ cell activation, causing a tumor-specific immune reaction. 
In vitro and in vivo experimental evidence of nsPEF causing immune reactions

Little is known regarding the induction of immune responses after in situ tumor destruction by nsPEF. It was hypothesized that nSPEF may induce membrane protein changes, which acting as "eat me" signals stimulate immune cells to attack the tumor. To test this, an in vitro co-culture experiment was set up using the macrophage cell line THP1 and HCC cells. When the nSPEF was delivered to the HCC cells, the macrophage cell line THP1 engulfed the nsPEF-treated HCC cells and not the HCC cells without nsPEF. ${ }^{14}$ The in vitro cell line experiment results were further supported by results from nude mice xenograft model experiments, which showed that local nsPEF inhibits primary HCC in liver and metastatic HCC in lungs. ${ }^{14}$

\section{nsPEF-induced immune reaction is transferable}

Other experiments have further indicated that the antitumor reaction can be transferred. ${ }^{15-17}$ Local nsPEF ablates the tumor and then the dying cancer cells release tumor antigens and immune recruiting signals, which serve to enhance the antitumor $\mathrm{T}$ cell responses to attack both primary tumors and distant metastases. This immune stimulation response may play a critical role in the nsPEF-elicited tumor eradication. Of note, it has been reported that nSPEF stimulates macrophages to engulf tumor cells in vitro and in vivo. ${ }^{13,14}$ The multiple dose strategy with fractured multiple treatments was also shown to induce immune defense against the tumor by triggering externalization of phosphatidylserine on the cell membrane; this serves as a vital signal to attract macrophages, neutrophils and dendritic cells for phagocytic clearance of the ablation area. $^{14}$

Such processes help to break the tolerance of the tumor, ${ }^{14}$ which is especially important for late-stage tumors with remote metastasis. Beebe et al. ${ }^{15}$ also found that animals with successfully ablated primary tumors failed to have secondary tumors emerge, due to the adaptive immune response.
The nsPEF-ablated location was shown to be infiltrated by immune cells and granzyme $B$, suggesting an immune-protective effect. Nuccitelli et $a l^{16,17}$ found that after complete ablation of HCC with nsPEF, a second tumor, which had been injected into a different lobe of the liver, was also inhibited by $90 \%$ via $\mathrm{CD} 8^{+}$cytotoxic T cells.

Local nsPEF ablation reshapes the local tumor microenvironment and decreases remote metastasis

Chen et al. ${ }^{18}$ investigated the antitumor effect of nsPEFs in two different in vivo tumor models with lung metastasis (i.e., spontaneous osteosarcoma and HCC); both models involved late stage and showed high metastatic potential. The nSPEF treatment reduced primary tumor volume and increased the total survival significantly, without deformity or thermal damage in the ablation zone. Immune cells were found in the ablation site of the tumor. ${ }^{13,19,20}$ Hematoxylin and eosin staining showed that the nsPEF-treated tumor shrank dramatically and then became surrounded by a remarkable infiltration of inflammatory cells.

High density of MAC387-positive cells was observed in perivascular areas, confirming that nsPEF treatment linked host immune surveillance, the organ self-defensive barrier and electric conductivity of different tissues in the ablation zone, reshaping the local tumor microenvironment. nsPEF caused microdomain disruption and increased the membrane permeability, facilitating further immune cell recognition and interaction. $^{20,21}$ The possible mechanism of the nsPEF-induced immune reaction is illustrated in Fig. 1.

\section{Discussion}

HCC ablation with nsPEF requires a special catheter electrode design and internal delivery by laparoscopy. Currently, there is no clinical study describing its application in human liver. Beside HCC, nsPEF has been applied to multiple solid tumors. For example, nsPEF has been shown to enhance the anti-tumor effects of the mTOR inhibitor everolimus against melanoma, ${ }^{22}$

nsPEF

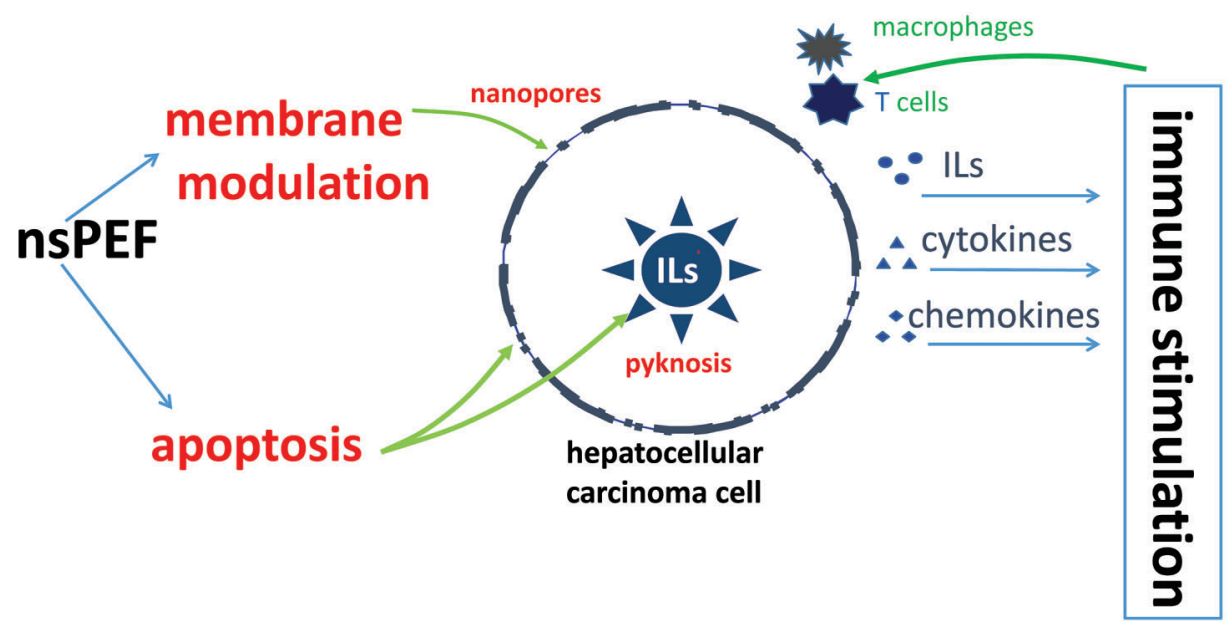

Fig. 1. Host immune responses induced by nanosecond pulsed electric field. 
to collapse vascular perfusion in glioblastoma, ${ }^{23}$ to inhibit proliferation in osteosarcoma, ${ }^{24}$ and to serve as breast cancer therapy ${ }^{25}$ and against salivary adenoid cystic carcinoma. ${ }^{26}$

When treating different solid tumors, application of nsPEF raises the questions of proper selection for dosage, parameters and strategies based on the tumor differences and patient personalization. Studies on cancer cell susceptibility have been done to address these issues. Gianulis et al. ${ }^{27}$ tested cytotoxic efficiency of nsPEF for different cancers and found no apparent correlation with cell types. Recent studies $^{28,29}$ showed that, beside nsPEF dosage, temperature is another important issue when applying nsPEF in practice. Pliquett et al. ${ }^{2}$ measured the real time Joule heating and proved that nSPEF ablation under a certain parameter (i.e. pulse duration of 300 ns and electric field strength of $40 \mathrm{kV} / \mathrm{cm}$ ) produced no obvious heat production or temperature increase. Mi et al. ${ }^{28}$ expanded the measurement to a multi-parametric setting (i.e. nsPEF parameter ranging from 1 to $4 \mathrm{kV}$, pulse width ranging from 50 to $500 \mathrm{~ns}$, and repetition frequency between $100 \mathrm{kHz}$ and $1 \mathrm{MHz}$ ). Their data indicated that higher temperatures will be achieved and may cause thermal damage when multiple pulse bursts are applied. These results collectively provide the theoretical basis of pulse parameter selection for future clinical parameter settings. Yin et al. ${ }^{29}$ also confirmed that environmental temperature can affect the outcome of nsPEF treatment.

\section{Conclusions}

In conclusion, nsPEF has been demonstrated as an efficient local ablation methodology for HCC treatment. By nano-pulse stimulation, nsPEF more efficiently inhibits tumors without increasing the risk of secondary metastasis. Beyond its ablation effect, nsPEF may elicit tumor cell death by stimulating immune defense. Therefore, nsPEF ablation alone or in combination with other immuno-therapeutics could be used as a locoregional therapy for HCC in clinic.

\section{Acknowledgements}

This study was funded by the Xinjiang Key Lab Project (No. 2014 KL002 to X Chen), the National Natural Science Foundation of China (No. 81372425 to X Chen), National S\&T Major Project (No. SQ2018ZX100301 to X Chen) and the Zhejiang Natural Science Foundation (No. LY17H160018 to X Miao).

\section{Conflict of interest}

The authors have no conflict of interests related to this publication.

\section{Author contributions}

Reviewed the literature and contributed equally to the study $(X X, Y C)$, contributed to reference collection (RZ), supervised the review $(X M, X C)$.

\section{References}

[1] Feng K, Ma KS. Value of radiofrequency ablation in the treatment of hepatocellular carcinoma. World J Gastroenterol 2014;20:5987-5998. doi: 10. 3748/wjg.v20.i20.5987.

[2] Pliquett $U$, Nuccitelli R. Measurement and simulation of Joule heating during treatment of B-16 melanoma tumors in mice with nanosecond pulsed electric fields. Bioelectrochemistry 2014;100:62-68. doi: 10.1016/j.bioelechem. 2014.03.001.

[3] Chen X, James Swanson R, Kolb JF, Nuccitelli R, Schoenbach KH. Histopathology of normal skin and melanomas after nanosecond pulsed electric field treatment. Melanoma Res 2009;19:361-371. doi: 10.1097/CMR. Ob013e32832f1558.

[4] Chen X, Kolb JF, Swanson RJ, Schoenbach KH, Beebe SJ. Apoptosis initiation and angiogenesis inhibition: melanoma targets for nanosecond pulsed electric fields. Pigment Cell Melanoma Res 2010;23:554-563. doi: 10.1111/ j.1755-148X.2010.00704.X.

[5] Nuccitelli R, Wood R, Kreis M, Athos B, Huynh J, Lui K, et al. First-in-human trial of nanoelectroablation therapy for basal cell carcinoma: proof of method. Exp Dermatol 2014;23:135-137. doi: 10.1111/exd.12303.

[6] Chen XH, Beebe SJ, Zheng SS. Tumor ablation with nanosecond pulsed electric fields. Hepatobiliary Pancreat Dis Int 2012;11:122-124.

[7] Nuccitelli R, Tran K, Sheikh S, Athos B, Kreis M, Nuccitelli P. Optimized nanosecond pulsed electric field therapy can cause murine malignant melanomas to self-destruct with a single treatment. Int J Cancer 2010;127:1727-1736. doi: $10.1002 / i j c .25364$.

[8] Beebe SJ. Cell responses without receptors and ligands, using nanosecond pulsed electric fields (nsPEFs). Int J Nanomedicine 2013;8:3401-3404. doi: 10.2147/IJN.S51357.

[9] Beebe S], Chen YJ, Sain NM, Schoenbach KH, Xiao S. Transient features in nanosecond pulsed electric fields differentially modulate mitochondria and viability. PLoS One 2012;7:e51349. doi: 10.1371/journal.pone.0051349.

[10] Beebe SJ, Sain NM, Ren W. Induction of Cell Death Mechanisms and Apoptosis by Nanosecond Pulsed Electric Fields (nsPEFs). Cells 2013;2:136-162. doi: $10.3390 /$ cells2010136.

[11] Ren Z, Chen X, Cui G, Yin S, Chen L, Jiang J, et al. Nanosecond pulsed electric field inhibits cancer growth followed by alteration in expressions of NF-кB and Wnt/ $\beta$-catenin signaling molecules. PLoS One 2013;8:e74322. doi: 10.1371 /journal.pone.0074322.

[12] Nuccitelli R, Chen X, Pakhomov AG, Baldwin WH, Sheikh S, Pomicter JL, et al. A new pulsed electric field therapy for melanoma disrupts the tumor's blood supply and causes complete remission without recurrence. Int J Cancer 2009;125:438-445. doi: 10.1002/ijc.24345.

[13] Chen X, Yin S, Hu C, Chen X, Jiang K, Ye S, et al. Comparative study of nanosecond electric fields in vitro and in vivo on hepatocellular carcinoma indicate macrophage infiltration contribute to tumor ablation in vivo. PLoS One 2014;9:e86421. doi: 10.1371/journal.pone.0086421.

[14] Yin S, Chen X, Hu C, Zhang X, Hu Z, Yu J, et al. Nanosecond pulsed electric field (nSPEF) treatment for hepatocellular carcinoma: a novel locoregional ablation decreasing lung metastasis. Cancer Lett 2014;346:285-291. doi: 10.1016/j.canlet.2014.01.009.

[15] Chen R, Sain NM, Harlow KT, Chen YJ, Shires PK, Heller R, et al. A protective effect after clearance of orthotopic rat hepatocellular carcinoma by nanosecond pulsed electric fields. Eur J Cancer 2014;50:2705-2713. doi: 10.1016/j. ejca.2014.07.006

[16] Nuccitelli R, Mallon Z, Berridge C, Kreis M, Athos, B, Nuccitelli P. Nanoelectroablation of tumors leads to the translocation of calreticulin to the cell surface and the initiation of immunogenic apoptosis. The 11th International Bioelectrics Symposium, Columbia, Missouri, USA, 2014:42.

[17] Nuccitelli R, Tran K, Lui K, Huynh J, Athos B, Kreis M, et al. Non-thermal nanoelectroablation of UV-induced murine melanomas stimulates an immune response. Pigment Cell Melanoma Res 2012;25:618-629. doi: 10. $1111 / j .1755-148 X .2012 .01027 . x$

[18] Chen X, Chen Y, Jiang J, Wu L, Yin S, Miao X, et al. Nano-pulse stimulation (NPS) ablate tumors and inhibit lung metastasis on both canine spontaneous osteosarcoma and murine transplanted hepatocellular carcinoma with high metastatic potential. Oncotarget 2017;8:44032-44039. doi: 10 . 18632/oncotarget.17178.

[19] Nuccitelli R, McDaniel A, Anand S, Cha J, Mallon Z, Berridge JC, et al. NanoPulse Stimulation is a physical modality that can trigger immunogenic tumor cell death. J Immunother Cancer 2017;5:32. doi: 10.1186/s40425-0170234-5.

[20] Yin S, Chen X, Xie H, Zhou L, Guo D, Xu Y, et al. Nanosecond pulsed electric field (nsPEF) enhance cytotoxicity of cisplatin to hepatocellular cells by microdomain disruption on plasma membrane. Exp Cell Res 2016;346: 233-240. doi: 10.1016/j.yexcr.2016.06.018.

[21] Kulbacka J. Nanosecond pulsed electric fields (nsPEFs) impact and enhanced Photofrin $\mathrm{II}(\circledR)$ delivery in photodynamic reaction in cancer and normal cells. Photodiagnosis Photodyn Ther 2015;12:621-629. doi: 10.1016/j.pdpdt. 2015.11.002.

[22] Dai J, Wu S, Kong Y, Chi Z, Si L, Sheng X, et al. Nanosecond pulsed electric fields enhance the anti-tumour effects of the mTOR inhibitor everolimus against melanoma. Sci Rep 2017;7:39597. doi: 10.1038/srep39597.

[23] Bardet SM, Carr L, Soueid M, Arnaud-Cormos D, Leveque P, O'Connor RP. Multiphoton imaging reveals that nanosecond pulsed electric fields collapse tumor and normal vascular perfusion in human glioblastoma xenografts. Sci Rep 2016;6:34443. doi: 10.1038/srep34443. 
[24] Miao X, Yin S, Shao Z, Zhang Y, Chen X. Nanosecond pulsed electric field inhibits proliferation and induces apoptosis in human osteosarcoma. J Orthop Surg Res 2015;10:104. doi: 10.1186/s13018-015-0247-z.

[25] Wu S, Guo J, Wei W, Zhang J, Fang J, Beebe SJ. Enhanced breast cancer therapy with nsPEFs and low concentrations of gemcitabine. Cancer Cell Int 2014;14:98. doi: 10.1186/s12935-014-0098-4.

[26] Qi W, Guo J, Wu S, Su B, Zhang L, Pan J, et al. Synergistic effect of nanosecond pulsed electric field combined with low-dose of pingyangmycin on salivary adenoid cystic carcinoma. Oncol Rep 2014;31:2220-2228. doi: 10.3892/or.2014.3063.
[27] Gianulis EC, Labib C, Saulis G, Novickij V, Pakhomova ON, Pakhomov AG. Selective susceptibility to nanosecond pulsed electric field (nSPEF) across different human cell types. Cell Mol Life Sci 2017;74:1741-1754. doi: 10. 1007/s00018-016-2434-4.

[28] Mi Y, Rui S, Li C, Yao C, Xu J, Bian C, et al. Multi-parametric study of temperature and thermal damage of tumor exposed to high-frequency nanosecondpulsed electric fields based on finite element simulation. Med Biol Eng Comput 2017;55:1109-1122. doi: 10.1007/s11517-016-1589-3.

[29] Yin S, Miao X, Zhang X, Chen X, Wen H. Environmental temperature affects physiology and survival of nanosecond pulsed electric field-treated cells. J Cell Physiol 2017. doi: 10.1002/jcp.25984 